\title{
NEURITE TRACING IN FLUORESCENCE MICROSCOPY IMAGES USING RIDGE FILTERING AND GRAPH SEARCHING: PRINCIPLES AND VALIDATION
}

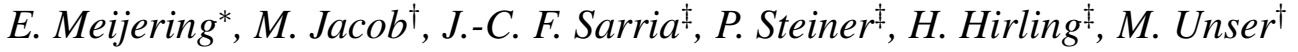 \\ ${ }^{*}$ Biomedical Imaging Group Rotterdam, Erasmus MC—University Medical Center Rotterdam \\ Depts. of Medical Informatics and Radiology, P. O. Box 1738, 3000 DR Rotterdam, The Netherlands \\ Email address: meijering@imagescience.org \\ ${ }^{\dagger}$ Biomedical Imaging Group, Swiss Federal Institute of Technology Lausanne \\ EPFL-STI-BIOE-LIB, BM-Ecublens, CH-1015 Lausanne, Switzerland \\ ${ }^{\ddagger}$ Laboratory of Cellular Neurobiology, Swiss Federal Institute of Technology Lausanne \\ EPFL-SV-INS-LNC, AI-Ecublens, CH-1015 Lausanne, Switzerland
}

\begin{abstract}
To assist neurobiologists investigating the molecular mechanisms involved in neurite formation and differentiation, we have developed an interactive technique for the tracing and quantification of elongated image structures. The technique is based on an improved steerable filter for computing local ridge strength and orientation. It also uses a graph-searching algorithm with a novel cost function exploiting these image features to obtain globally optimal tracings between userdefined control points. To compare the performance of the technique to that of the currently used approach of fully manual delineation, four observers traced selected neurites in fluorescence microscopy images of cells in culture, using both methods. The results indicated that the proposed technique yields comparable accuracy in measuring neurite length, significantly improved accuracy in neurite centerline extraction, significantly improved reproducibility and reduced user interaction.
\end{abstract}

\section{INTRODUCTION}

The development of the nervous system is an intricate process involving many different factors. Studies into the molecular mechanisms regulating neurite formation and differentiation often involve extensive image analysis. A task of particular importance is the tracing and quantification of the neurites emanating from the cell body, which is often done by tedious manual delineation. In the past decade, only a handful of more advanced neurite tracing techniques have been published. Recent examples, developed for 3D confocal microscopy image stacks, are based on vectorial tracking [1] and multiscale feature analysis [2].

In this paper we are concerned with 2D fluorescence microscopy images of individual cells. Whereas the computational cost of neurite tracing can be expected to be smaller in $2 \mathrm{D}$ than in $3 \mathrm{D}$, the task is hampered by ambiguities due to the crossing of neurites. In addition, neurites may have very low contrast or contain gaps. And when compiled from several scans, the images may show background intensity discontinuities or gradients (Fig. 1). Procedures based on thresholding $[3,4]$ are likely to fail in such cases. Some authors have resorted to heuristic approaches $[5,6]$. Another recently published technique [7] was successfully applied to images of retinal explants, but does not address the mentioned problems seen in our type of images.

Based on these facts, we conclude that there is still a great need for image analysis tools facilitating neurite tracing. The design of fully automated tracing techniques will remain a difficult problem, however, and it seems likely that some form of user interaction will always be required to resolve ambiguities. In this paper we describe a novel, interactive neurite tracing technique and present the results of validation experiments revealing its performance compared to fully manual delineation, in terms of accuracy, reproducibility, and required user interaction.

\section{TECHNIQUE}

Neurite tracing using our technique consists of (i) a detection phase and (ii) a linking phase. The detection algorithm is based on the fact that in the images, neurites are bright, elongated structures superimposed on a dark, noisy background. It is known $[8,9]$ that such structures are well analyzed by second-order operators. Improving on existing approaches, we compute for every pixel $\mathbf{x}$ in the image

$$
\mathbf{H}_{f * G}^{\prime}(\mathbf{x})=\mathbf{H}_{f * G}(\mathbf{x})+\alpha \mathbf{R}_{\pi / 2}^{T} \mathbf{H}_{f * G}(\mathbf{x}) \mathbf{R}_{\pi / 2},
$$

where $\mathbf{H}_{f * G}$ denotes the Hessian of the Gaussian-smoothed version of the image, and $\mathbf{R}_{\pi / 2}$ the matrix representing a rotation with angle $\pi / 2$. Computing the eigenvalues of (1) 


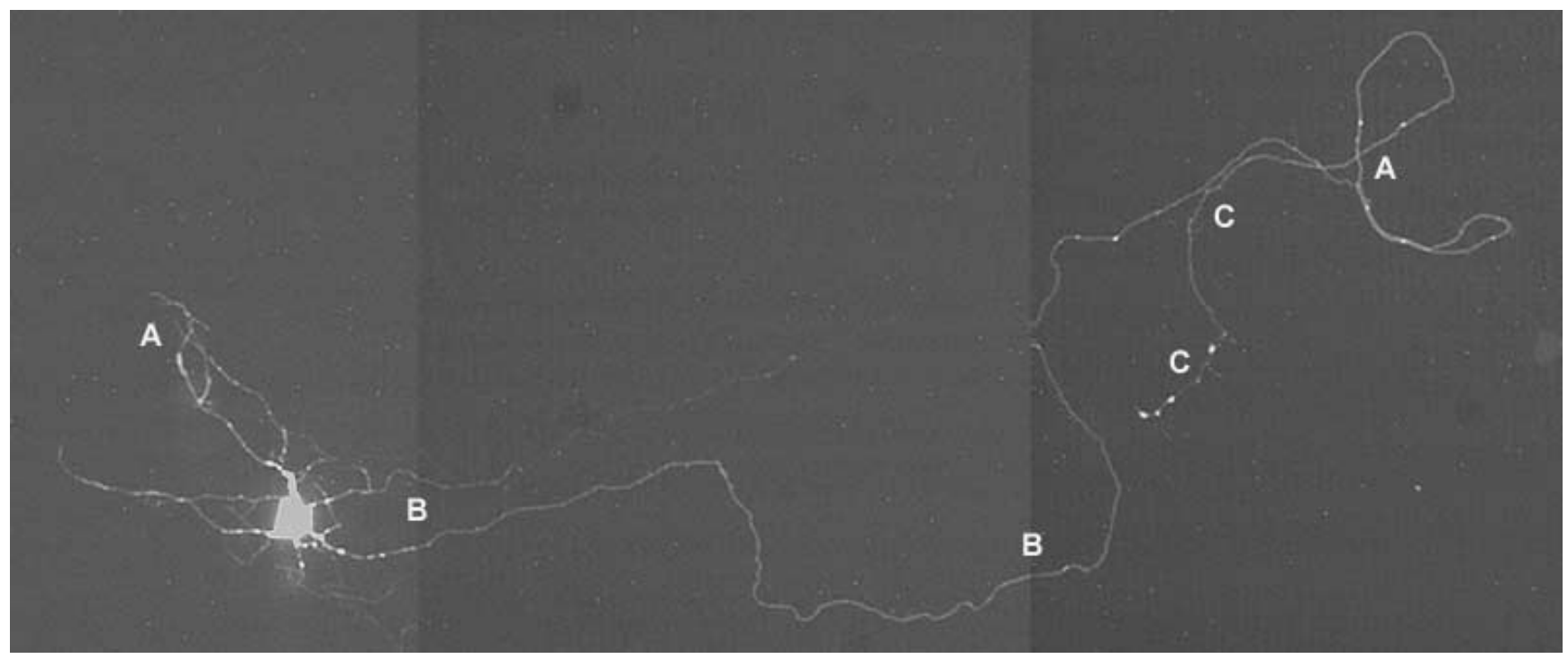

Fig. 1. Part of a fluorescence microscopy image showing a hippocampal neuron in culture. The image was compiled from different scans to capture all of the neuron's outgrowth. It illustrates the different problems encountered in neurite tracing, such as ambiguities caused by the branching or crossing of neurites (as in the regions indicated by 'A'), background intensity discontinuities or gradients (as in regions ' $\mathrm{B}$ '), and varying or even discontinuous neurite contrast (compare regions ' $\mathrm{C}$ ').

is equivalent to computing the maximum and minimum response to the steerable filter $\mathbf{r}_{\theta}^{T} \cdot \mathbf{H}_{G}^{\prime} \cdot \mathbf{r}_{\theta}$, with $\mathbf{r}_{\theta}$ the unit vector in direction $\theta$. Taking $\alpha=-1 / 3$ in (1) implies a filter (Fig. 2) that is maximally flat in the direction $\theta+\pi / 2$. As a measure of neurite strength we then compute

$$
\rho(\mathbf{x})= \begin{cases}\lambda(\mathbf{x}) / \lambda_{\min } & \text { if } \quad \lambda(\mathbf{x})<0, \\ 0 & \text { if } \quad \lambda(\mathbf{x}) \geq 0,\end{cases}
$$

where $\lambda$ is the larger in magnitude of the two eigenvalues and $\lambda_{\text {min }}$ denotes the smallest $\lambda$ over all pixels. Also stored for each pixel is the local neurite orientation as indicated by $\mathbf{v}$, the normalized eigenvector corresponding to the smaller absolute eigenvalue (Fig. 3). Since the Gaussian kernel involved in the computations is a scalable function, with scale parameter $\sigma$, the detector is tunable to neurites of specific width, while it also suppresses noise.

For the linking of consecutive pixels we have adopted the live-wire segmentation paradigm $[10,11]$. The new function that we propose to compute the cost of moving from any pixel $\mathbf{x}$ to an eight-connected pixel $\mathbf{y}$ is

$$
\mathrm{C}(\mathbf{x}, \mathbf{y})=\gamma \mathrm{C}_{\lambda}(\mathbf{y})+(1-\gamma) \mathrm{C}_{\mathbf{v}}(\mathbf{x}, \mathbf{y}),
$$

where $\gamma \in[0,1]$ is a parameter determining the relative weight of the two normalized cost components, $\mathrm{C}_{\lambda}$ and $\mathrm{C}_{\mathbf{v}}$. The former of these is computed from the eigenvalues as

$$
\mathrm{C}_{\lambda}(\mathbf{y})=1-\rho(\mathbf{y})
$$

and the latter from the eigenvectors as

$$
\mathrm{C}_{\mathbf{v}}(\mathbf{x}, \mathbf{y})=\frac{1}{2}\{\sqrt{1-\varphi(\mathbf{x}, \mathbf{y})}+\sqrt{1-\varphi(\mathbf{y}, \mathbf{x})}\},
$$

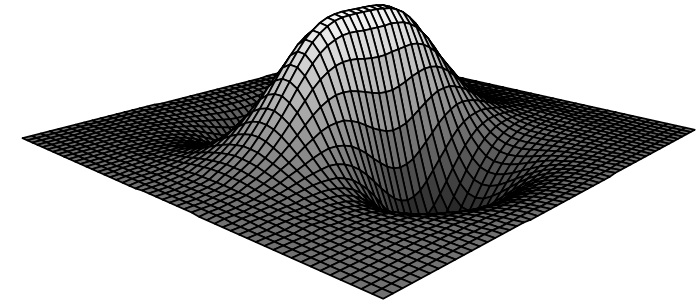

Fig. 2. Shape of the steerable ridge filter $\mathbf{r}_{\theta}^{T} \cdot \mathbf{H}_{G}^{\prime} \cdot \mathbf{r}_{\theta}$ implicitly used in the neurite detection algorithm. The filter, depicted here as an inverted landscape, is more elongated than the filter normally found in the literature on ridge detection.

where $\varphi(\mathbf{x}, \mathbf{y})=|\mathbf{v}(\mathbf{x}) \cdot \mathbf{d}(\mathbf{x}, \mathbf{y})|$, with $\mathbf{d}(\mathbf{x}, \mathbf{y})$ the unit vector in direction $\mathbf{y}-\mathbf{x}$. Optimal paths based on this cost function are computed by a fast implementation of Dijkstra's shortest-path algorithm $[10,11]$. The placing of starting points is facilitated by applying "local snapping" in a small window of $w \times w$ pixels around the actual cursor position. Smooth tracings (Fig. 4) are obtained by application of a uniform postfilter, of size $2 p+1$, to the path pixel coordinates, followed by subsampling with a factor $s$.

\section{VALIDATION}

After initial experimentation with the technique, the different parameters were fixed to $\sigma=2.0, \gamma=0.7, w=9$, $p=5$, and $s=5$. The technique was compared to fully manual tracing with a standard piecewise straight-line drawing technique. For the validation we selected 20 representative cell images from a previous study [12]. Four observ- 

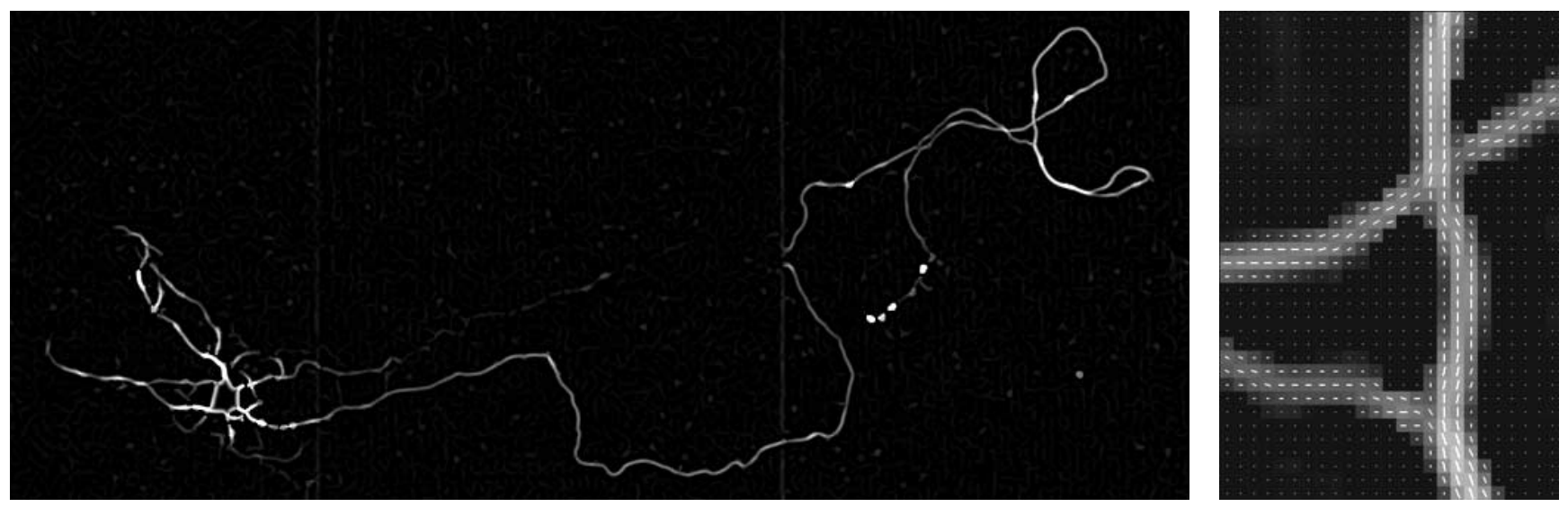

Fig. 3. Output of the neurite detection algorithm applied to the image in Fig. 1. The left image shows for every pixel the local neurite strength. The right image shows the computed local neurite orientations (superimposed white dashes) for the region indicated by the right-most 'A' in Fig. 1. Due to the use of second-order image features, the algorithm suppresses background intensity discontinuities and gradients. In addition, it is tunable to neurites of specific width and reduces the influence of noise.

ers independently traced selected neurites three times using both techniques. The motto was to minimize the number of mouse clicks while attaining sufficient tracing accuracy, according to the observers' own visual judgment. The accuracy of the tracings was determined by comparison with reference tracings, obtained by manual delineation in very high-resolution versions of the images, where the observers were asked to be as accurate as possible, regardless of the number of clicks required. Two different error measures were used: length difference and average deviation. For each of the tracings, the values for the two error measures were computed for each of the observers' corresponding reference tracing, and the resulting four error values per measure were averaged to yield a consensus error for that measure. The variability in the consensus errors was computed both per observer and between observers. Finally, the labor intensiveness of our technique versus fully manual tracing was analyzed by comparing the number of mouse clicks required per tracing. The statistical significance of the outcome of all comparisons was determined by means of a two-sided paired Student $t$-test, under the null hypothesis that the two techniques would give similar results.

\section{DISCUSSION}

From the results (Fig. 5) we observe that our semiautomatic neurite tracing technique yields a significant improvement over fully manual tracing methods in terms of true neurite centerline representation, reproducibility, and reduced user interaction. Adding the observation that the two perform comparably in the case of length measurements, we conclude that our technique may replace the latter. Due to the use of a global optimization algorithm and second-order image feature analysis, it is very robust against noise, back-
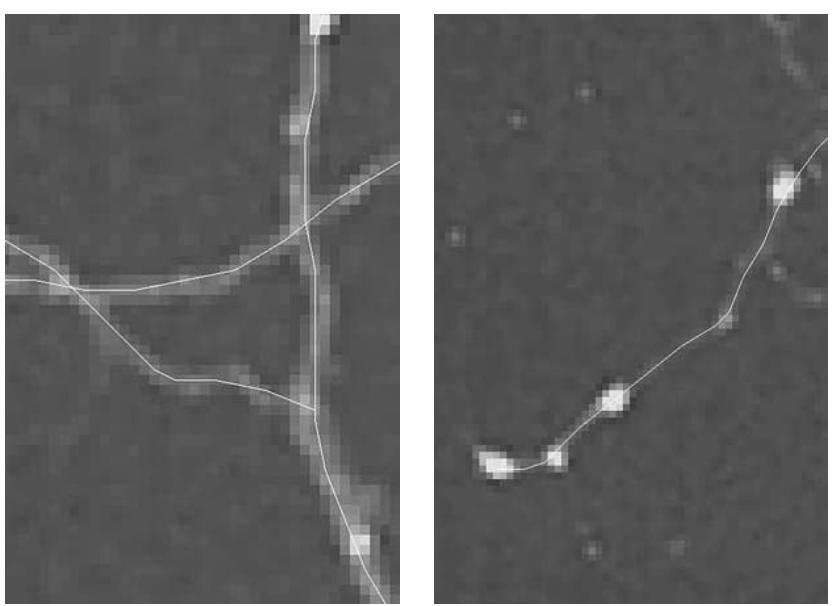

Fig. 4. Sample tracings (superimposed white curves) obtained with the described technique. The images show the results for the regions indicated by the right-most ' $\mathrm{A}$ ' (left image) and the lower ' $\mathrm{C}$ ' (right image) in Fig. 1 and demonstrate the power of the technique even in the case of poor neurite contrast.

ground intensity discontinuities or gradients, and varying or even locally diminishing neurite contrast. As a result it can be applied to a wide range of images without changing its parameters. The number of parameters to be set by the user is small. A disadvantage of the technique is that it still requires user interaction, which limits its applicability to images containing manageable amounts of neurites only. The challenge for future research will be, therefore, to further automate the process by computing landmarks to serve as input for the optimization algorithm. A logical approach would be to choose high-curvature and branch points for this purpose. In the meantime, the technique is already being applied successfully in our institutes. 

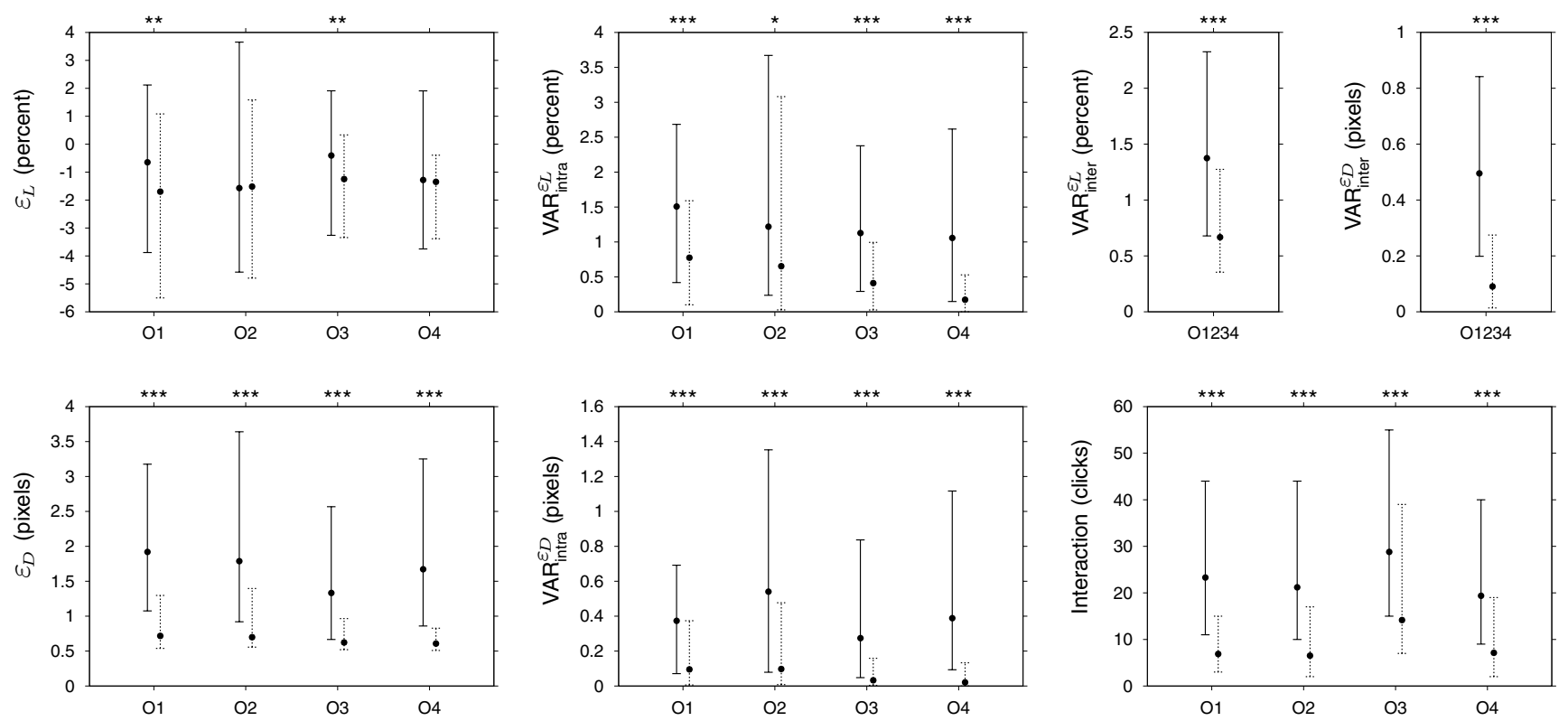

Fig. 5. Results of the validation experiments comparing fully manual tracing (solid bars) and the described semiautomatic tracing technique (dotted bars). The graphs show the observed values for the length difference $\left(\varepsilon_{L}\right)$ and the average deviation $\left(\varepsilon_{D}\right)$ measure, the intraobserver variability for both measures $\left(\mathrm{VAR}_{\text {intra }}^{\varepsilon_{L}}\right.$ and $\left.\mathrm{VAR}_{\text {intra }}^{\varepsilon_{D}}\right)$, the interobserver variability for both $\left(\mathrm{VAR}_{\text {inter }}^{\varepsilon_{L}}\right.$ and $\left.\mathrm{VAR}_{\text {inter }}^{\varepsilon_{D}}\right)$, and the amount of user interaction required. The bars show the full range of observed values (with the black circles indicating the mean values) separately for each of the observers (O1-O4). The asterisks on top of each graph indicate for each observer the probability for the null hypothesis of equivalence to be true based on the data presented. The different levels of probability considered are $p<0.001(* * *), p<0.01(* *)$, $p<0.05(*)$, and any probability larger than 0.05 (no asterisk).

\section{REFERENCES}

[1] K. A. Al-Kofahi, S. Lasek, D. H. Szarowski, C. J. Pace, G. Nagy, J. N. Turner, and B. Roysam, "Rapid automated three-dimensional tracing of neurons from confocal image stacks," IEEE Trans. Inf. Technol. Biomed., vol. 6, pp. 171$187,2002$.

[2] A. Dima, M. Scholz, and K. Obermayer, "Automatic segmentation and skeletonization of neurons from confocal microscopy images based on the 3-D wavelet transform," IEEE Trans. Image Process., vol. 11, pp. 790-801, 2002.

[3] E. R. A. Jap Tjoen San, M. H. Schmidt-Michels, B. M. Spruijt, A. B. Oestreicher, P. Schotman, and W. H. Gispen, "Quantitation of the growth-associated protein B-50/GAP-43 and neurite outgrowth in PC12 cells," J. Neurosci. Res., vol. 29, pp. 149-154, 1991.

[4] B. Malgrange, P. Delrée, J. M. Rigo, H. Baron, and G. Moonen, "Image analysis of neuritic regeneration by adult rat dorsal root ganglion neurons in culture," J. Neurosci. Methods, vol. 53, pp. 111-122, 1994.

[5] R. Ventimiglia, B. E. Jones, and A. Møller, "A quantitative method for morphometric analysis in neuronal cell culture: Unbiased estimation of neuron area and number of branch points," J. Neurosci. Methods, vol. 57, pp. 63-66, 1995.

[6] L.C.B. Rønn, I. Ralets, B. P. Hartz, M. Bech, A. Berezin, V. Berezin, A. Møller, and E. Bock, "A simple procedure for quantification of neurite outgrowth based on stereological principles," J. Neurosci. Methods, vol. 100, pp. 25-32, 2000.

[7] C. M. Weaver, J. D. Pinezich, W. B. Lindquist, and M. E. Vazquez, "An algorithm for neurite outgrowth reconstruction," J. Neurosci. Methods, vol. 124, pp. 197-205, 2003.

[8] Y. Sato, S. Nakajima, N. Shiraga, H. Atsumi, S. Yoshida, T. Koller, G. Gerig, and R. Kikinis, "Three-dimensional multi-scale line filter for segmentation and visualization of curvilinear structures in medical images," Med. Image Anal., vol. 2, pp. 143-168, 1998.

[9] A. F. Frangi, W. J. Niessen, R. M. Hoogeveen, T. van Walsum, and M. A. Viergever, "Model-based quantitation of 3D magnetic resonance angiographic images," IEEE Trans. Med. Imaging, vol. 18, pp. 946-956, 1999.

[10] E. N. Mortensen and W. A. Barrett, "Interactive segmentation with intelligent scissors," Graph. Models Image Process., vol. 60, pp. 349-384, 1998.

[11] A. X. Falcão, J. K. Udupa, and F. K. Miyazawa, “An ultrafast user-steered image segmentation paradigm: Live wire on the fly," IEEE Trans. Med. Imaging, vol. 19, pp. 55-62, 2000.

[12] P. Steiner, J. C. F. Sarria, B. Huni, R. Marsault, S. Catsicas, and H. Hirling, "Overexpression of neuronal sec1 enhances axonal branching in hippocampal neurons," Neuroscience, vol. 113, pp. 893-905, 2002. 